\title{
16 \\ GAME MECHANICS, EXPERIENCE DESIGN, AND AFFECTIVE PLAY
}

Patrick Jagoda and Peter McDonald

This chapter explores games as a major object of study in both media theory and practice. We begin by identifying two competing critical approaches to games: the proceduralist (which emphasizes rules, objectives, and systems) and the play-centric (which emphasizes modes of player response). By distinction, we advocate for a middle ground, a type of experience design that foregrounds the ways players can affect and be affected by a game: experientially, kinesthetically, and ideologically. The main site for this elaboration of affect is game mechanics. The chapter draws from close readings of existing digital and analog games, as well as techniques developed through the creative process in the Game Changer Chicago (GCC) Design $\mathrm{Lab}$, to which we both belong. Ultimately, we offer a sketch of a practice-based research method for designing learning-oriented and serious games in media studies.

\section{The Procedural and the Playful}

Two primary approaches have dominated game studies since the early 2000s, one organized around procedure and the other around play. In an important study of storytelling in computational environments, Janet Murray observes that a key attribute of computers is that they "execute a series of rules" that enable dynamic interactions between the machine and human actors (1997: 71-72). Storytellers and game designers, Murray contends, can use algorithms and rules to convey interpretations. One of the most vocal theorists of proceduralism, Ian Bogost, defines it as a way of generating and making sense of processes. Processes, in turn, "define the way things work: the methods, techniques, and logics that drive the operation of systems, from mechanical systems like engines to organizational systems like high schools to conceptual systems like religious faith" (Bogost 2007: 3). Videogames are a medium that uses, and even depends on, what Bogost calls "procedural rhetoric," which "explains processes with other processes" in order to persuade users of a particular viewpoint (9). This procedural approach privileges a game's rules and its representation of select systems.

For example, the single-player, turn-based videogame PeaceMaker (Impact Games, 2007), puts the player in the perspective of either the Israeli prime minister or the Palestinian 
president. PeaceMaker produces a system in which the player's success hinges on a multifaceted political ecology. Actions reveal implicit ethical arguments: delivering peaceful speeches or establishing welfare programs are more consistently rewarded with higher scores than militaristic responses. Even more explicitly, the game posits a two-state solution as its goal. PeaceMaker does not produce an expository argument about responses to international gridlock (as a blog post might) nor does it craft a psychological perspective on this system (as a stream-of-consciousness novel might). Instead, the game invites players to experiment with algorithmic processes to better understand a set of political processes, as modeled by the designers.

Proceduralism has contributed to the analysis of videogames as cultural objects and influenced design practices, especially of so-called "serious games" that explicitly address social and political topics in the interest of participatory advocacy. At the same time, scholars more interested in processes of play than game structures have emphasized the limitations of procedural rhetoric as an analytic and design technique. At the forefront of the antiprocedural approach is Miguel Sicart, who contends that proceduralism is essentially a version of formalism. A consequence of proceduralism, Sicart argues, is that "it grants great power and influence to the designer," who produces the system that the player must, in turn, reverse engineer sufficiently to unpack the meaning that is inherent in it (2011). For Sicart, the limitation of proceduralism is that it marginalizes the ways in which people play by stipulating that meaning largely precedes the act of play. In other words, a game's procedural rhetoric belongs to its system of rules and objectives, rather than to the ways in which players test and transform that system. In contrast, play-centric accounts demonstrate that actions and meanings cannot prefigure play. As Alexander Galloway argues, though machine actions are key, digital games only "exist when enacted" (2006: 2). This focus on play has found proponents not only in the humanities, but also among sociology and anthropology scholars who use qualitative techniques, such as interviews, to study a plurality of player interpretations (Voorhees 2013).

In videogames, different players may indeed have distinct experiences of action, narrative, or roleplaying. For example, Kurt Squire argues that open-ended simulation or "sandbox" games, such as Civilization III and Grand Theft Auto: San Andreas, operate as "possibility spaces" in which players can "try on, inhabit, and ultimately develop new identities with trajectories for participation that extend out of the game world and into new spaces" (2008: 171-2). In other cases, a game's artificial intelligence may promote emergent gameplay by adapting dynamically to a player's skill and present situation. The "AI Director" of Left 4 Dead works collaboratively with players to produce dramatic encounters and spawns "Infected" enemies based on player performance, creating novel play scenarios. Even in games that are not created explicitly to promote emergent play styles, participants frequently use a system in ways that were not intended or predicted by the designers. For instance, players may find creative ways to exploit glitches, such as "rocket jumping" in Quake or Halo, that allow them to achieve greater elevation by (mis)using a weapon for mobility. In still other cases, games themselves produce variable experiences from session to session through procedurally generated characters (e.g., creatures in Spore) and levels (e.g., the fantasy world of Dwarf Fortress).

This theoretical rift is roughly parallel to the earlier split in literary criticism between, on the one hand, Russian Formalism and American New Criticism and, on the other hand, reader-response theory and deformative criticism that privileged creative interpretation. To be fair, however, procedural and play-centric theories are not mutually exclusive. Most proceduralists do, to some extent, account for the role of play in game activities. For example, Bogost notes: 
In a procedural representation like a videogame, the possibility space refers to the myriad configurations the player might construct to see the ways the processes inscribed in the system work. This is really what we do when we play videogames: we explore the possibility space its rules afford by manipulating the game's controls.

(Bogost 2007: 42)

In this formulation, seemingly inexpressive processes can still enable complex forms of expression. Even so, Sicart (2014) is right that meaning is, for Bogost, founded in procedures whereas, for him, procedures are secondary to the possibilities of play.

\section{Experience Design: Rules, Mechanics, and Affect}

Sicart goes as far as to argue that "games don't matter" when compared to the more generalized processes of play unfolding in toys, playgrounds, technologies, and social groups (2014: 2). Though we share Sicart's investment in a broader landscape of play, as game designers we are also interested in what both procedural and play-centric theories have to teach us about game creation practices. Given the increasingly practice-based orientation of digital humanities and new media studies, especially as they are taken up in this volume, we would like to use these frameworks to think about the design of serious games. One bridge between procedures and play through design comes from Katie S. Tekinbaş and Eric Zimmerman (2003). As they show, game designers may start by creating a rule-based system and defining some of its affordances, but the success of a game ultimately depends on experience design that privileges the prospective player. As Tekinbas puts it, a designer becomes "a socio-technical engineer, thinking about how people will interact with the game and how the game will shape both competitive and collaborative social interaction" (2007: 305). Instead of over-determining form, this orientation yields an evocative design created for ambiguous and open-ended experiences.

The concept of experience design suggests an internal tension insofar as it combines the control of design with the uncontrollable diversity of player experiences. Thus, design may influence experience, but only in an indirect way. Even so, rather than limiting playfulness, Tekinbaş and Zimmerman argue that rules "create multiple levels of play experience, layering strategic thinking and gradual skill acquisition on top of the physical and perceptual components of the core mechanic" (2003: 320). Game mechanics are the set of techniques for interacting with a game world that are arbitrarily mapped through an interface to player gestures and are both constrained and enabled by a game's platform, whether it is a PlayStation 4 console or an analog game board. These actions, expressed as verbs, might include "jumping" in a platformer or "targeting" in a first-person shooter. For Tekinbaş and Zimmerman, and for us, a mechanic is a key point of mediation between the player and the game that opens up the ambiguity of play. A mechanic provides a point of departure from which a variety of procedural rhetorics can proliferate. On the one hand, a mechanic is a kind of rule, an action that the game can allow or disallow; on the other, players must take up that act as their own for the game to proceed.

Mechanics arguably provide the clearest example of how constraint imposed through design can enable creativity and playfulness instead of limiting players. For example, Super Mario World opens up pleasurable kinesthetic sensations and bodily identifications by allowing its players to fly. However, the sense of freedom in flying only emerges against the backdrop of Mario's ordinary activities of running and jumping. Reciprocally, because flight requires a power-up and takes a significant running start, and because its duration depends on a player's 
skill, Mario's repertoire of jumps accumulates meanings. It might be required to traverse a level, or it might enable the enjoyment of acrobatic movements. The point we want to emphasize, contra Sicart and others, is that rules are not essentially prohibitive (as Michel Foucault already argues of rules in a broader socio-historical context). Instead, they multiply possible meanings of play by creating new contexts of action.

If the meanings of a game are negotiable, and emerge from the interplay between rules and experience, between procedure and play, then mechanics may serve to orient both game analysis and design. While game mechanics may not yield the kind of discursive argumentation of procedural rhetoric, they constitute an ideal unit of analysis for affective experiences. Though it has many connotations, the term "affect" refers essentially to a "nonconscious experience of intensity" that differs from either feelings that are personal and narrativizable or emotions that are visible and communicative (Shouse 2005). As verbs, mechanics help us think about potentials, transformations, desires, intensities, experimental engagements, and connections among players. Importantly, while the process of narrating game experiences often entails describing affects as feelings, mechanics do not merely produce unified results in which jumping elicits one kind of bodily sense whereas targeting evokes another. Jumping provokes myriad feelings, including elation, freedom, curiosity, or a sense of precarity, while targeting produces malice, satisfaction, the sense of appropriative connection to a target, or a feeling of bodily extension through a world. More than defining feelings, mechanics enable players to affect the world of the game, and in turn be affected by it, opening up aleatory experiences. We are using affect here as somewhat synonymous with feeling, but also want to draw on the legacy of Spinoza for whom the possible activity and passivity of a body determines its feelings. Gilles Deleuze observes of Spinoza that "we do not even know what a body can do," and this is equally true of the gamer's or the avatar's body (1983: 36). Fundamentally, mechanics can generate proliferating affects and meanings that exceed a one-toone signification.

As we have been discussing them, mechanics mediate between rule-bound situations and player affects. As Tekinbaş and Zimmerman put it:

To play a game is to experience the game: to see, touch, hear, smell, and taste the game; to move the body during play, to feel emotions about the unfolding outcome, to communicate with other players, to alter normal patterns of thinking.

(Tekinbaş \& Zimmerman 2003: 314)

Though "fun" is perhaps the most frequently invoked aspect of game experience, games generate a wide range of affects. Some of these may serve persuasive purposes, but others are more ambiguous or aesthetic in nature. Procedural analyses often limit the discussion of affective experiences, restrict them to oversimplified typologies, or characterize them as ideological elements. Similarly, discussions of a game's "interactivity" often privilege agency, figured as choice that a player does or does not have. However, beyond the binary split implicit in fun or agential interactivity, players may also experience affects such as anxiety, paranoia, anticipation, and curiosity in the process of encountering hardware, interfaces, game worlds, and player cultures.

In the remaining sections, we begin to sketch out the ways that game mechanics might open up more nuanced forms of analysis and design strategies. To do so, we focus neither on a priori rules, objectives, and systems (as proceduralists might) nor modes of play (as play-centric theorists might). Instead, charting a middle path, we explore the meaning of mechanics in play, first in game analysis and second in game design. 


\section{The Case of "Collecting" in Katamari Damacy: Game Analysis}

The three interpretive methods that we are comparing-practices that focus on rules (proceduralism), play (play-centrism), and mechanics (experience design)—can usefully be juxtaposed by showing how they operate in the analysis of a single game. The mechanic of "collecting" in the innovative videogame, Katamari Damacy (Namco 2004; see Figure 16.1), serves as a clarifying case. In this game, players use two analog sticks to control "The Prince," an alien avatar who rolls around a sticky ball that collects everything in its path. This "katamari" ball grows from an initial size of $5 \mathrm{~cm}$ (at which point it collects tacks and ants) to an eventual size of over 300 meters (at which point the katamari can collect cars and buildings). In each level, the katamari must reach a certain size within a time limit. In addition to the assorted mass, players collect special objects that form larger collections such as cousins of the Prince and uniquely named versions of ordinary objects. New media scholars McKenzie Wark (2007) and Steven Jones (2008) offer readings of the game that privilege procedure and play, respectively. In contrast, we offer a third reading that describes a range of affects that the game activates through collecting.

Wark's Gamer Theory (2007) articulates a strong version of the proceduralist thesis. For Wark, it is because a world framed by late capitalism has adopted the structure of games that this medium can help us understand the present. Particular games make aspects of capitalism easier or more difficult to see through their procedural rhetoric, or what Wark (following Alexander Galloway's neologistic combination of "allegory" and "algorithm") calls their "allegorithm." Even so, the simplest game already contains the defining qualities of capitalism. In Wark's reading, Katamari Damacy becomes an updated myth of Sisyphus, and an allegory for the capture of the analog by the digital. For instance, the game transforms the aleatory line of the katamari into thresholds of size and time that convert it into a binary value of

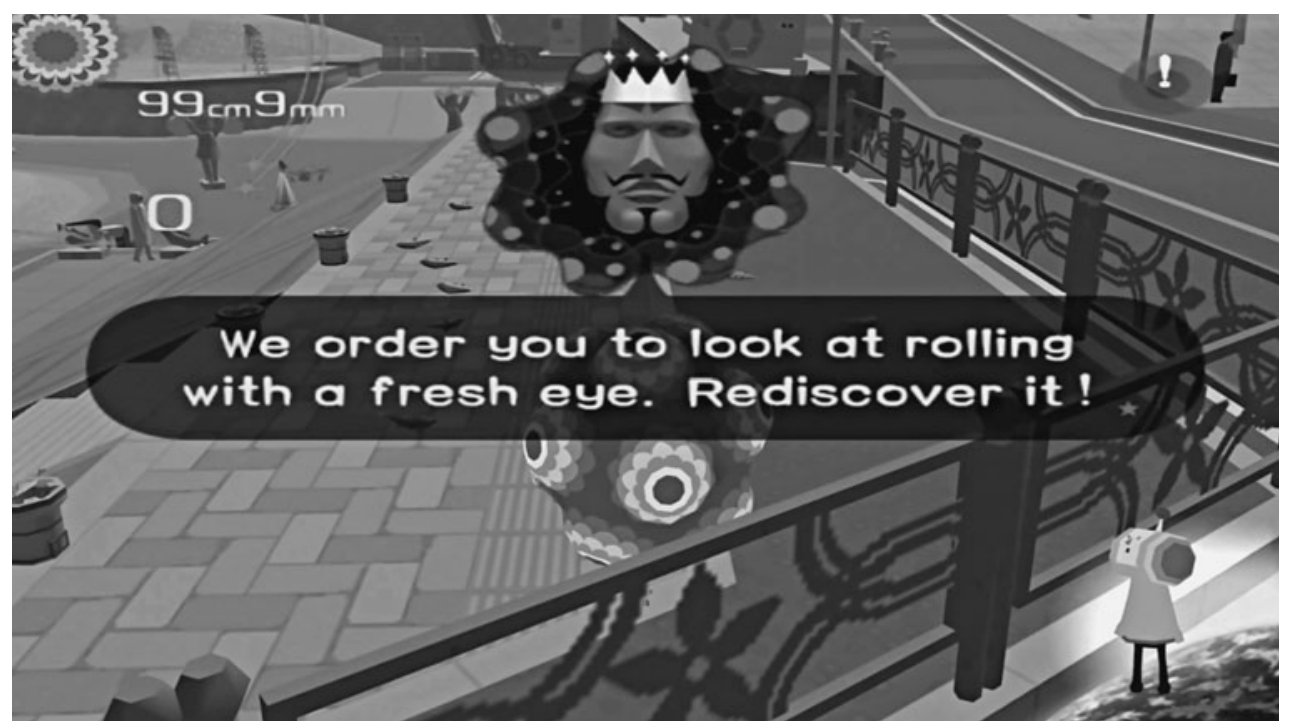

Figure 16.1 Katamari Damacy.

Source: Namco 2004. 
success or failure, removing the older ambiguity of Sisphysus' task. The omnivorous tendency of the katamari to absorb even those things that appear solid, such as the walls that demarcate a level's boundaries, demonstrates the reduction of qualitative difference to numerical quantities. The katamari also operates across radically different scales, substituting a human perspective for the microscopic and the cosmic. This summary is not an exhaustive list of what Wark suggests we can see through Katamari Damacy, but the point is that in each case the specific procedural argument can be translated into a statement, a fact that we now know about late capitalism.

In an essay that is in part a response to Wark, Steven Jones also argues that Katamari Damacy tells us something essential about games. Instead of treating collecting as a symptom of capitalism, Jones characterizes this mechanic as a feature of most games in which a player picks up a weapon or a key and generates meaning through that process. In this way, collecting in a videogame is an act that is comparable to collecting on eBay or during archival research. Jones takes Katamari Damacy to embody a punk aesthetic in its anarchic collecting mechanic that evacuates pre-established meaning and leaves a void that demands to be filled by player actions. Because it has no content of its own, collecting in this game is "inherently an activity that points outside of itself, outside of the game itself . . because it only truly has meaning in the context of a fanbase or other kind of community" (Jones 2008: 64). The playful path of the ball, the qualities of the objects that are rolled together, the kinesthetic pleasure of the joysticks - these are the elements from which players generate meaning.

For both Wark and Jones, Katamari Damacy is a privileged example because the game reflects on its material and formal conditions. In our own approach, one which privileges the way that mechanics generate affects, Katamari Damacy is also important because it operates as a tour de force of collecting, exploring the various senses of the verb that are visible in more isolated forms in games such as Pokémon or Go Fish. Unlike the real world collections that Jones explores, the objects in Katamari Damacy are ideal in a variety of senses that alter the feeling of collecting them. For instance, they do not degrade with time, they cannot be destroyed, and they are not removed from circulation when placed in another player's collection. Collecting is thus removed in this game from an involvement in an economy, making rare acquisitions correspond directly to virtuosic performance in the game. Desire is indexed to difficulty rather than rarity. Furthermore, because objects cannot be destroyed, the potential for growth matters more than the threat of loss, a feeling made visceral whenever players recklessly knock pieces off their katamari ball.

In Katamari Damacy, collecting is recontextualized by rules that idealize the world. The mechanic responds with affordances of fearlessness, virtuosic performance, and yearning. In this structure of feeling, we might recognize both the Sisyphean labor of Wark's account and the anarchic punk aesthetic of Jones. But there are other valences as well that can illuminate thematic choices within the game, such as the narrative resonance with courtly love or an alternate relation to the father that bypasses the anxiety of castration or loss. The meaning of collecting is not unitary, nor tied to the rhetorical purposes of a particular game, but neither is it vague or characteristic of all play. A mechanic constellates an array of feelings, a palette that a designer or player can reliably draw upon.

\section{The Case of "Blocking" in Smoke Stacks: Game Design}

Affects are key to game experience in general, but they play an especially central role in learning-oriented games. Though affect as such does not figure prominently in literature about 
educational games, related discussions about emotion inform practice-based research in digital humanities and new media studies. James Paul Gee argues that work in education has traditionally emphasized rationality and intellectual content over emotion. However, as he adds, emotion helps learners to integrate previous knowledge and organize long-term memory, focus attention and retrieve information, and evaluate possible actions (2008: 35). Games that seek to promote learning, then, must be attentive to the range of possible play experiences, including gradations of various named emotions and nonconscious intensities.

Since the 1980s, many educational games-including what Mizuko Ito calls "academic" games such as Math Blaster and "entertainment" games such as Where in the World is Carmen Sandiego? (2008: 89-116) — have offered interactive content delivery without a robust emotional experience. Similarly, serious games with social and political objectives sometimes put a greater emphasis on conveying values through game systems in a direct fashion, as Mary Flanagan and Helen Nissenbaum have described the work of the "conscientious designer" (2014: xii-xiii). While content and values are important in such games, emotions and especially obscure or illegible affects can sometimes become marginal.

The analysis of the collecting mechanic that we suggested in the previous section demonstrates the types of affects, feelings, and moods that games make available. An understanding of how core game mechanics generate such affects, and the meanings that follow from them, may be even more useful for designing games that are meant to tackle specific social issues. At the GCC Design Lab, we create both digital and board games that explore issues of social and emotional health related to topics such as unplanned pregnancy and bullying. In designing such games, we often experiment with the affective affordances of mechanics through techniques of rapid prototyping and iterative design. Because our goal is often to influence the knowledge, attitudes, or behaviors of players, an understanding of affect becomes especially important.

To briefly demonstrate how mechanics and affects are important to serious game design, we take up a case study of a board game that the authors created as part of the GCC Design Lab. This game, Smoke Stacks, tackles the use of tobacco products among high school youth. The game is one in a suite designed for the same Hexacago board, which depicts the city of Chicago broken down into hexagons. The learning objectives for Smoke Stacks include helping players understand marketing approaches deployed by the tobacco industry and their impact on smoking behaviors. By helping youth understand the history of tobacco legislation as well as the health effects of smoking, the game attempts to curb interest in tobacco products. In the game, four to six players take the role of tobacco companies who try to accumulate as many customers as possible. In each round, players formulate corporate strategies by bidding on cards that provide them with products (e.g., "cigarettes" or "chewing tobacco"), marketing approaches (e.g., making customers feel "independent" or "sexy"), and advertising media (e.g., "television" or "billboards"). These cards allow each player to target particular customers in certain zones on the board, while every customer increases a player's profit margin.

In creating Smoke Stacks, we did not privilege a uniform play experience but rather attended to the broad range of affects that mechanics might generate. The three primary mechanics in this game are collecting (picking up customers from particular urban zones), wagering (on corporate strategy cards), and blocking (keeping other players from collecting the cards they want). In our iterative design process, we were attentive, for example, to the affordances of blocking. In our research on blocking in games, we analyzed sports games, popular board games such as Settlers of Catan, and tower defense videogames. Across these genres, we characterized blocking as a mechanic that arises through the player's investment in a project that is under threat. In many of these games, players often go to wasteful lengths to negate a threat 
and regain a sense of safety, and they give up only when the situation feels utterly futile. To block, the player must understand the motives of an opponent and the details of the game system. Such understanding infuses minute actions with a sense of urgency, while sometimes generating feelings of pettiness, aggression, and desire for revenge.

In the Smoke Stacks design, mechanics foreground specific feelings that attend political, economic, and social systems often imagined at scales beyond individual human experience. In other words, mechanics make the abstraction of systems tangible. In the context of tobacco use, blocking gives players an opportunity to work through the affective dimensions of how certain policies may lead to a competitive race to the bottom. Players can block other players from either collecting desired cards or accumulating the maximum number of customers. The experience of blocking another player animates ways in which inequality is built into policies, even when the rationality of large-scale systems such as "the market" is meant to overshadow human motivations and feelings.

The tobacco system that Smoke Stacks models allows a player to experiment with elements of corporate strategy, profit motives, addictive consequences, and histories of government legislation. The game makes this affective field, which includes both systemic and historical elements, accessible and palpable through mechanics. Given the complexity of this dynamic system, the process of experience design required frequent playtesting with ongoing player feedback and designer self-reflection over several months. An iterative design method maximized the affordances of the core mechanics. Thus, rather than seeking uniform reception, the design process explored the possible experiences that each version of the game made possible.

\section{Conclusion}

This chapter offers only a sketch of a form of game analysis and experience design that privileges mechanics as a way to understand the affective dimensions of broader systems. Although we focused on a few case studies, experience design is important to a wide array of methods across games and genres. Such methods might include not only close reading but also the study of online archives of player responses to games, focus groups and interviews with players, and practice-based research that includes creating and play testing games to better understand their affordances. The field of game studies, and perhaps especially the growing area of serious game analysis and design, will benefit from a fuller understanding of the relationship between mechanics and affects.

\section{Further Reading}

Bogost, I. (2006) Unit Operations: An Approach to Videogame Criticism, Cambridge, MA: MIT Press.

Flanagan, M. (2009) Critical Play: Radical Game Design. Cambridge, MA: MIT Press

Isbister, K. (2016) How Games Move Us: Emotion by Design, Cambridge, MA: MIT Press.

Juul, J. (2005) Half-Real: Video Games Between Real Rules and Fictional Worlds, Cambridge, MA: MIT Press.

Miller, K. (2012) Playing Along: Digital Games, YouTube, and Virtual Performance, Oxford: Oxford University Press.

Wardrip-Fruin, N. (2009) Expressive Processing: Digital Fictions, Computer Games, and Software Studies, Cambridge, MA: MIT Press.

\section{References}

Bogost, I. (2007) Persuasive Games: The Expressive Power of Videogames, Cambridge, MA: MIT Press.

Deleuze, G. (1983) Nietzsche and Philosophy, New York, NY: Columbia University Press.

Flanagan, M. and H. Nissenbaum (2014) Values at Play in Digital Games, Cambridge, MA: MIT Press. 
Galloway, A. (2006) Gaming: Essays on Algorithmic Culture, Minneapolis, MN: Minnesota University Press.

Gee, J. P. (2008) "Learning and Games," in K. S. Tekinbaş (ed.) The Ecology of Games: Connecting Youth, Games, and Learning, Cambridge, MA: MIT Press.

Ito, M. (2008) "Education vs. Entertainment: A Cultural History of Children's Software,” in K. S. Tekinbaş (ed.)

The Ecology of Games: Connecting Youth, Games, and Learning, Cambridge, MA: MIT Press.

Jones, S. E. (2008) The Meaning of Videogames, New York, NY: Routledge.

Murray, J. (1997) Hamlet on the Holodeck: The Future of Narrative in Cyberspace, New York, NY: The Free Press.

Shouse, E. (2005) "Feeling, Emotion, Affect," M/C Journal 8.6.

Sicart, M. (2011) "Against Procedurality," Game Studies 11.3.

Sicart, M. (2014) Play Matters, Cambridge, MA: MIT Press.

Squire, K. (2008) "Open-Ended Video Games: A Model for Developing Learning for the Interactive Age," in K. S. Tekinbaş (ed.) The Ecology of Games: Connecting Youth, Games, and Learning, Cambridge, MA: MIT Press.

Tekinbaş, K. S. (2007) “Gaming Literacies: A Game Design Study in Action,” Journal of Educational Multimedia and Hypermedia 16.3.

Tekinbaş, K. S. and E. Zimmerman (2003) Rules of Play: Game Design Fundamentals Cambridge, MA: MIT Press. Voorhees, G. (2013) "Criticism and Control: Gameplay in the Space of Possibility," in M. Wysocki (ed.) Ctrl-AltPlay: Essays on Control in Video Gaming, Jefferson, NC: McFarland.

Wark, M. (2007) Gamer Theory, Cambridge, MA: Harvard University Press. 